\title{
Ultrastructural Pathology of Plasma and Endoplasmic Reticulum Membranes of Nerve and Glial Cells: A Review
}

\author{
Orlando J Castejon* \\ Biological Research Institute, Venezuela

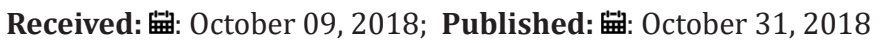 \\ *Corresponding author: Orlando J Castejon, Biological Research Institute, Venezuela
}

\begin{abstract}
The alteration of nerve cell plasma membranes is reviewed in some neuropathological conditions. In moderate brain oedema a continuous plasma membrane is observed but the cytoplasmic membranes, such as smooth and rough endoplasmic reticulum membranes appear damaged. In severe oedema, fragmentation of plasma membrane, enlargement and focal necrosis of rough endoplasmic cisterns and nuclear envelope, detachment of membrane-bound ribosomes, and reduction of polysome are found. Shallow and deep invaginations of plasma membrane, and the formation of endocytic and clathrin-coated vesicles are seen. In astrocyte cells, areas of focal necrosis and fragmented limiting plasma membrane, overdistended rough endoplasmic reticulum cisterns with extense degranulated membrane domains, and vacuoles of smooth endoplasmic reticulum with necrotic limiting membrane are observed. Oligodendroglia cells show notably edematous changes featured by lacunar enlargement of rough endoplasmic reticulum and nuclear envelope, detachment of membrane bound ribosomes, and discontinuous plasma membrane. Plastic changes and damage of synaptic membranes are found.
\end{abstract}

Synaptic vesicle exocytosis and endocytosis at the non-specialized regions of presynaptic ending limiting membrane are frequently observed at activated synapses. In severe brain edema, synaptic disassembly occurs featured by wide separation of pre and postsynaptic membranes, and loss of peri synaptic astrocytic glial escheatment. Disruption, fusion and disassembly of interastrocytary gap junctions have also been observed. The endothelial cell luminal membrane of brain capillaries undergoes profound activity changes that characterize increased cerebrovascular permeability, such as increased formation of micro- and macropinocytotic vesicles, clathrin coated vesicles, and emission of pseudopods to form endothelial vacuoles. The alterations of nerve cell plasma membranes and cytomembranes are related with the anoxic-ischemic conditions of brain parenchyma. The role of free radical and lipid peroxidation, disturbed energy metabolism, altered metabolic cascades, glutamate excitotoxicity, haemoglobin toxicity, protein aggregation, and presence of extracellular oedema fluid are discussed in relation with the derangement of nerve cells membranes.

Abbreviations: Endoplasmic Reticulum (ER), Prion Protein (Prp), Parkinson's Disease (PD), Amyotrophic Lateral Sclerosis (ALS), Unfolded Protein Response (UPR), Mitochondria-Associated ER Membranes (Mams) Alzheimer's Disease (AD), Vesicle-Associated Membrane Protein-Associated Protein B (VAPB), Protein Tyrosine Phosphatase-Interacting Protein 51 (PTPIP51), Nitric Oxide Synthase (NOS), Mitochondria (M), Neural Cell Adhesion Molecules (NCAM)

\section{Introduction}

Over the four past decades substantial progress have been made in elucidating the mechanisms by which nerve plasma membranes and cytomembranes are damaged. Nerve cell membrane alterations were earlier described by Bass [1] in Creutzfeldt- Jacob disease. Significant enrichment in cholesterol ester was found by [2] In nerve cell membranes in adrenoleukodystrophy. Disorganization of endoplasmic reticulum cisterns, formation of lamellar bodies, and hypertrophy of Golgi complex were described by Malunova and Samoilov [3] in cat cerebral cortex as early post-anoxic changes. Tissue surrounding hematomas, traumatic lesions, infective zones, and certain tumours undergo autocatalytic peroxidation
[4], a lipidic disorder which greatly altered membrane functions. Demyelination-induced plasticity was found by Coria [5] in the nodal and internodal axolemma in rat lead-induced neuropathy. Nuclear membrane indentations were reported by Roos [6] in Huntington's disease.

Biochemical and morphological changes in nerve cell membranes were reported by Salvati [7] in experimental allergic encephalomyelitis. Macromolecular structure of axonal membrane during acute experimental allergic encephalomyelitis, and myelin deficient rat optic nerve was described by Black [8] and Waxman [910] found a drop-in cholesterol content in neuronal plasmalemma 
after cerebral ischemia. [11] encountered continuities between the outer nuclear membrane and the rough endoplasmic reticulum in hyppocampal neurons during seizure-induced protein synthesis. Membrane damage during situations of acute or subacute cerebral aggression have been studied using experimental models [4,1216]. Glial membrane damage of glial axonal junction after diffusing axonal injury was reported by Maxwell.

Traumatic brain injuries produce damage to nodal axolemma [17] and a widespread derangement to the neuronal cytoskeleton $[18,19]$ delayed phospholipids degradation [20], and calpainmediated spectr in breakdown leading to ischemic neuronal death. Babu [21] reported several protein defects in the plasma membrane of neurons and astrocytes in chronic ethanol treated rats. Dux [22] described disaggregation of ribosomes as an early sign of histotoxic pathology heralding delayed neuronal death. Theriault described gap junction remodelling in the rat spinal cord after acute compression injury. Maxwell [23] found alterations in the axolemma and myelin sheath in guinea pig optic nerve after stretch injury. Praproknit [24] described plasma membrane fragility in dystrophic neuritis of senile plaques in Alzheimer's disease. Dabrowska-Bouta $[25,26]$ showed alteration of myelin membranes after chronic lead intoxication in rats.

Torp [27] described association of fibrillar beta amyloid with neuronal membrane surface in aged dog brains. Similar findings were also reported by Yamaguchi [28] in hereditary cerebral haemorrhage with amyloidosis-Dutch type, Alzheimer disease, and non-demented aged subjects. Haik demonstrated that the putative transmembrane domain of prion protein induces neurotoxicity and destabilize nerve cell membranes.

\section{Brain Ischemia and Membrane Damage}

Glutamate ecotoxicity-induced damage of plasma membrane occurs in transient global cerebral ischemia [29] and in traumatic brain injuries [30] Peroxidative damage to cell membranes occurs following cerebral ischemia [31-35]. Transient global cerebral ischemia triggers suppression of protein synthesis, a process controlled by endoplasmic reticulum function [36-38]. Protein aggregation examined by electron microscopy and laser-scanning confocal microscopy has been reported after focal brain ischemia. It has been suggested that nitric oxide may contribute to ischemiainduced cell injury acting upon endoplasmic reticulum, calcium homeostasis, protein synthesis, and energy metabolism [39].

Damaged of nerve cell plasma membranes, cytoskeleton, rough and smooth endoplasmic reticulum membranes, lysosomal limiting membrane, and outer and inner mitochondrial membranes have been reported by [41-43] in moderate and severe edema associated to congenital hydrocephalus, brain trauma and brain tumors. Recent studies have suggested that cholesterol, an important component of membranes that controls their physical properties and functions, plays a critical role in neurodegenerative diseases. Enrichment of neuronal plasma membrane with cholesterol protects cortical neurons from apoptosis induced by soluble oligomers of the Abeta (1-40) peptide. Conversely, cholesterol depletion renders cells more vulnerable to the cytotoxic effects of the Abeta soluble oligomers [44]. The binding of Abeta to membrane lipids facilitates Abeta fibrillation, which in turn disturbs the structure and function of membranes, such as membrane fluidity or the formation of ion channels [45].

Recent reports also indicate that dysfunction of endoplasmic reticulum, which not only mediates proteins processing, but also regulates intracellular calcium homeostasis and cell death signal activation, occurs at an early stage after ischemia, and might be the initial step of apoptotic cascades in neurons [46]. Singleton and Povlishock [47] reported plasma membrane disruption in diffuse brain injury. Luo and Shi [48] have found that acrolein, a by-product of oxidative stress and lipid peroxidation, inflicts severe axolemmal disruption. The membrane damage is likely mediated by reactive oxygen species and lipid peroxidation, which are elevated after acrolein exposure. Shi has also reported axolemmal disruption in guinea pig spinal cord following compression. Kurnellas [49] have described plasma membrane calcium ATPase deficiency in multiple sclerosis and spinal cord injury, as a potential mechanism of neurodegeneration. Farkas [50] demonstrated mechanoporation or disruption of neuronal plasma membrane induced by diffuse traumatic brain injury.

Thompson [51] demonstrated opening of neuronal gap junction hemichannels following ischemia after stroke. Yi have found an increase in complexing I and complexing II, considered respectively markers of inhibitory and excitatory synapses, after traumatic brain injury. Nitric oxide and its toxic metabolite, peroxynitrite, can inhibit components of the mitochondrial respiratory chain leading to cellular energy deficiency and, eventually, to cell death. Kynurenine metabolic pathway, its alterations and their potential association with cellular energy impair certain neurodegenerative diseases. During energy production, most of the 02 consumed by the mitochondria is reduced fully to water, but $1-2 \%$ of the 02 is reduced incompletely to give the superoxide anion 02- If the function of one or more respiratory chain complexes is impaired for any reason, the enhanced production of free radicals further worsens the mitochondrial function by causing oxidative damage to macromolecules, and by opening the mitochondrial permeability transition pores thereby inducing apoptosis [52]. Prion protein (PrP), normally a cell surface protein, has been detected in the cytosol of a subset of neurons. The appearance of PrP in the cytosol could result from either retro-translocation of misfolded PrP from the endoplasmic reticulum (ER) or impaired import of PrP into the ER [53].

\section{Endoplasmic Reticulum Stress and Mitochondria Interplay and the Pathogenesis of Neurodegeneration}

Endoplasmic reticulum and mitochondria are in a close communication, establishing a dynamic ER-Ca2+-mitochondria interconnection that can play a prominent role in the neuronal cell death induction under stressful circumstances of Parkinson's disease pathology (PD). Also, endoplasmic reticulum (ER) stress in conjunction with abnormal protein degradation can contribute 
to the PD pathophysiology, [54]. Endoplasmic reticulum stress, initiated by the accumulation of unfolded or misfolded proteins, activates the unfolded protein response, which adapts cells to the stress. If this adaptive response is insufficient, the unfolded protein response activates an apoptotic program to eliminate the affected cells- Endoplasmic reticulum stress in myelinating cells is important in the pathogenesis of various disorders of myelin, including Charcot-Marie-Tooth disease, Pelizaeus-Merzbacher disease and Vanishing White Matter Disease, as well as in the most common myelin disorder, multiple sclerosis [55].

Amyotrophic lateral sclerosis (ALS) is a devastating neurodegenerative disease characterized by the misfolding and aggregation of distinct proteins in affected tissues, however, the pathogenic cause of disease remains unknown. Recent evidence indicates that endoplasmic reticulum (ER) stress plays a central role in ALS pathogenesis. ER stress activates the unfolded protein response (UPR), a homeostatic response to misfolded proteins. The UPR is initially protective by up-regulation of specific ER stressregulated genes and inhibition of general protein translation. However, long-term ER stress leads to cell death via apoptotic signaling, thus providing a link to neurodegeneration [56]. $\alpha$-synuclein pathology and its effects on diverse protein partners and specific cellular pathways in the membrane and/or cytosolic districts, such as endoplasmic reticulum/Golgi, axonal and synaptic compartments of dopaminergic neurons, may cause the onset of neuronal cell dysfunction and degeneration which are among the key pathological features of the Parkinson's disease (PD) brain. Besides, $\alpha$-synuclein aggregation may induce dysfunction and degeneration of synapses via these multiple mechanisms [57].

According to Hedskog [58], it is well-established that sub compartments of endoplasmic reticulum (ER) are in physical contact with the mitochondria. These lipid raft-like regions of ER are referred to as mitochondria-associated ER membranes (MAMs), and they play an important role in, for example, lipid synthesis, calcium homeostasis, and apoptotic signaling. Perturbation of MAM function has previously been suggested in Alzheimer's disease (AD). Our data suggest an important role of ER-mitochondria contacts and cross-talk in AD pathology.

$\alpha$-Synuclein is located in mitochondrial-associated endoplasmic reticulum membranes. PD-related mutated $\alpha$-synuclein results in its reduced association with mitochondria-associated membranes, coincident with a lower degree of apposition of endoplasmic reticulum with mitochondria and an increase in mitochondrial fragmentation. Aging is associated to cognitive decline and susceptibility to neuron death, two processes related recently to subcellular Ca2+ homeostasis. Memory storage relies on mushroom spines stability that depends on store-operated Ca2+ entry (SOCE). In addition, $\mathrm{Ca} 2+$ transfer from endoplasmic reticulum (ER) to mitochondria sustains energy production but mitochondrial $\mathrm{Ca} 2+$ overload promotes apoptosis. We have addressed whether SOCE and ER-mitochondria Ca2+ transfer is influenced by culture time in long-term cultures of rat hippocampal neurons, a model of neuronal aging [59].
However, many neuronal functions damaged in Parkinson's disease are regulated by signaling between the endoplasmic reticulum (ER) and mitochondria. This signaling involves close physical associations between the two organelles that are mediated by binding of the integral ER protein vesicle-associated membrane protein-associated protein $\mathrm{B}$ (VAPB) to the outer mitochondrial membrane protein, protein tyrosine phosphatase-interacting protein 51 (PTPIP51). VAPB and PTPIP51 thus act as a scaffold to tether the two organelles. $\alpha$-synuclein induced loosening of ER-mitochondria contacts is accompanied by disruption to $\mathrm{Ca} 2+$ exchange between the two organelles and mitochondrial ATP production. Such disruptions are likely to be particularly damaging to neurons that are heavily dependent on correct Ca2+ signaling and ATP [60].

\section{The Role of Calcium Overload}

Potentially toxic cytoplasmic calcium concentrations can also occur due to release from internal stores, either through physical damage to mitochondria and the endoplasmic reticulum, or a malfunction of receptors and channels present in their membranes. Such increases of cytoplasmic calcium concentrations can trigger a range of downstream neurotoxic cascades, including the uncoupling mitochondrial electron transfer from ATP synthesis, and the activation and overstimulation of enzymes such as calpains and other proteases, protein kinases, nitric oxide synthase (NOS), calcineurin and endonucleases [61].

\section{Autophagy and Apoptosis}

Autophagy and apoptosis are basic physiologic processes contributing to the maintenance of cellular homeostasis. Autophagy encompasses pathways that target long-lived cytosolic proteins and damaged organelles. It involves a sequential set of events including double membrane formation, elongation, vesicle maturation and finally delivery of the targeted materials to the lysosome. Deregulation of autophagy plays a pivotal role in the etiology and/ or progress of many neurodegenerative disorders [62]. Neurons employ specialized mechanisms to modulate local gene expression in dendrites, via the dynamic regulation of micro RNA biogenesis factors at intracellular membranes of the endoplasmic reticulum, which in turn is crucial for neuronal dendrite complexity and therefore neuronal circuit formation and function.

In the present review we describe the ultrastructural alteration of nerve cell and glial plasma membrane, cytomembranes, synaptic membranes, and interastrocytary gap junctions in cortical biopsies of patients with vascular anomalies, congenital hydrocephalus, complicated brain traumatic injuries. The brain parenchyma of these patients exhibits moderate and severe oedema and sustained anoxic-ischemic conditions [63-66].

\section{Alterations of Plasma Membrane and Cytomembranes in Vascular Anomaly and Moderate Oedema}

In vascular anomaly, the perifocal cerebral cortex exhibits a microstructure like that observed in normal animal brain cortex. The plasma membrane of non-pyramidal neurons shows in 
moderate oedema atly normal and continuous structure. The plasma membrane form endocytic and clathrin coated vesicles internalizing toward the cytoplasm. The limiting plasma membrane of rough endoplasmic reticulum cisterns exhibit fragmented areas and detachment of membrane associated ribosomes. The nuclear envelope appears irregularly dilated (Figures $1 \& 2$ ). Disaggregation of ribosomes was earlier demonstrated by Dux in primary cortical and hippocampal neuronal cultures after brief histotoxic hypoxia.

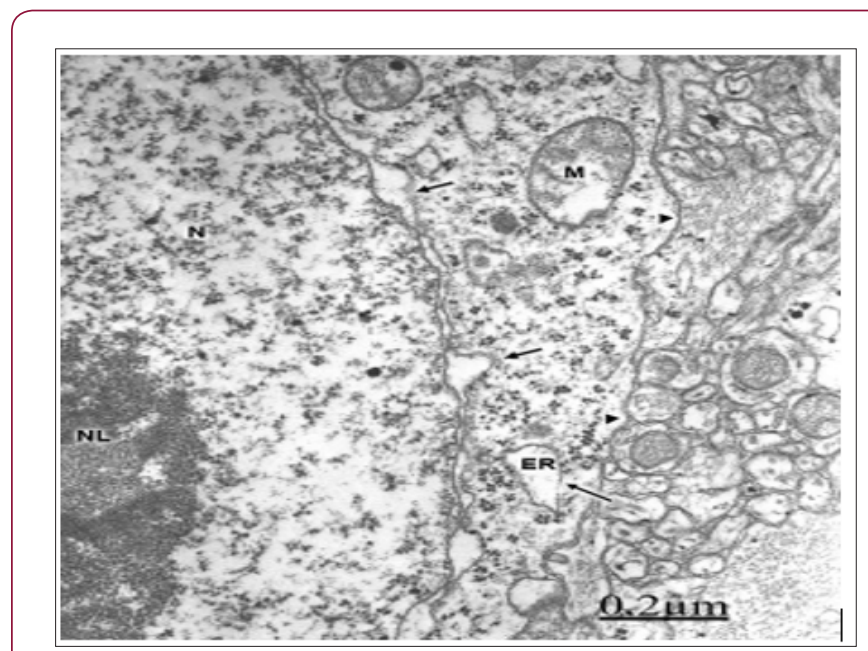

Figure 1: Anomaly of anterior cerebral artery. Right parietal cortex. Non-pyramidal nerve cell showing an irregularly dilated nuclear envelope (short arrows), enlarged endoplasmic reticulum cisterns (ER), detachment of membrane associated ribosomes (long arrows), and a continuous limiting plasma membrane (arrowheads). Note the prominent nucleus $(\mathrm{N})$, the nucleolus $(\mathrm{NL})$, the swollen mitochondria $(\mathrm{M})$, and the non-dilated extracellular space in the neighboring neuropil that features moderate brain edema.

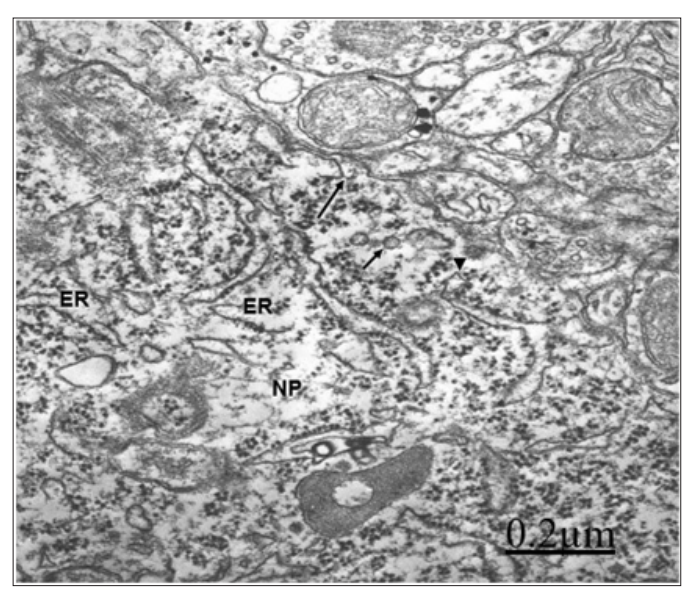

Figure 2: Anomaly of anterior cerebral artery. Right parietal cortex. Non-pyramidal neuron (NP) showing shallow invaginations of plasma membrane (long arrow) and formation of clathrin coated vesicle (arrowhead). Endocytic vesicles are also observed in sublemmal localization short arrows). Note the disrupted and degranulated rough endoplasmic reticulum cisterns (ER).

\section{Membrane Abnormalities in Congenital Hydrocephalus}

In relationship with congenital hydrocephalus in neonate patients, the presence of interstitial oedema fluid in the enlarged extracellular space of an immature neuropil induces fragmentation of plasma membrane, lacunar enlargement of rough endoplasmic reticulum, detachment of membrane associated ribosomes, irregularly dilated nuclear envelope, and nuclear pore disassembly [67] (Figure 3). Congenital hydrocephalus. Right frontal cortex. Non-pyramidal neuron exhibiting fragmented plasma membrane (long arrow), distended endoplasmic reticulum cisterns (ER), detachment of membrane associated ribosomes (short arrows), and disassembly of some nuclear pores (arrowheads). The mitochondria (M) appear swollen. Note the enlarged extracellular space in the neighbouring neuropil (asterisks). X 60.000.

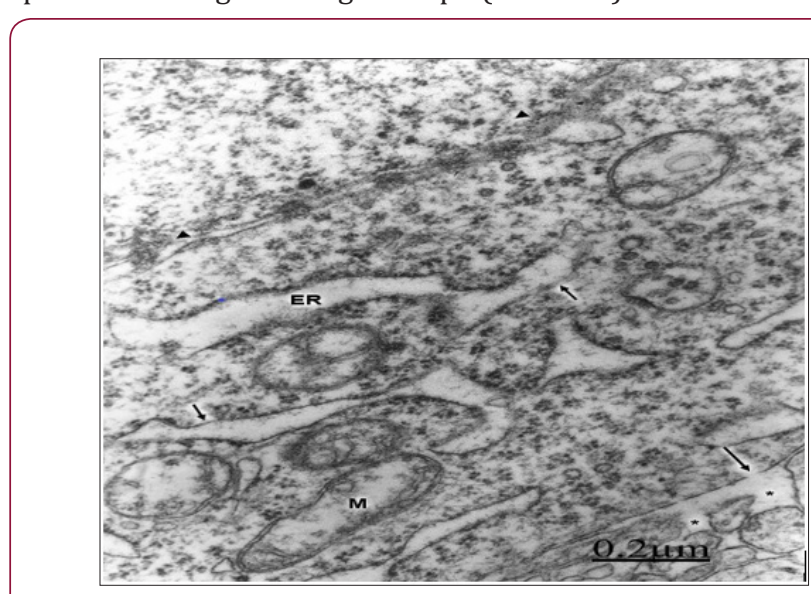

Figure 3: Congenital hydrocephalus. Right frontal cortex. Non-pyramidal neuron exhibiting fragmented plasma membrane (long arrow), distended endoplasmic reticulum cisterns (ER), detachment of membrane associated ribosomes (short arrows), and disassembly of some nuclear pores (arrowheads). The mitochondria (M) appear swollen. Note the enlarged extracellular space in the neighbouring neuropile (asterisks). X 60.000 .

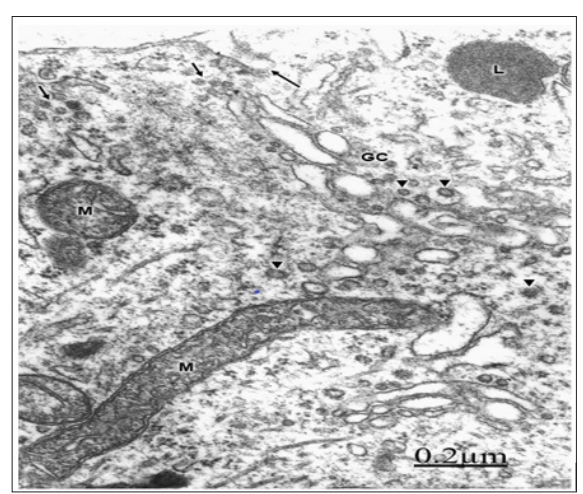

Figure 4: Congenital hydrocephalus. Right parietal cortex. The plasma membrane exhibits a deep invagination (long arrow) and formation of endocytic vesicles (short arrows) directed toward the swollen Golgi complex (GC). A lysosome (L), moderately swollen and dark mitochondria (M), numerous Golgi vesicles and clathrin-coated vesicles (arrowheads) also are seen. $X 60.000$. 
The plasma membrane shows deep invaginations and formation of numerous endocytic vesicles directed toward the endoplasmic reticulum and the Golgi apparatus [68]. The lysosomes show fragmented limiting membrane (Figure 4). In congenital hydrocephalus in neonate patients we are dealing with immature plasma membranes, characterized by changes in the integral membrane proteins, cholesterol domains, and in certain carbohydrates residues and anionic sites [69]. These different molecular compositions of immature plasma membranes explain its high sensitivity to injury factors. In hypertensive congenital hydrocephalus, the pressure exerted by the non-circulating cerebrospinal fluid induces plasma membrane fragmentation, and enlargement of intracellular nerve cell compartments. In addition, the presence of oedematous mitochondria evinces a disturbed energy metabolism.

Endocytosis is a fundamental mechanism by which neurons control intercellular signal, nutrient uptake, and synaptic transmission [70]. Clathrin-coated vesicles of different sizes have been isolated from rat brain, and related with the content of $\mathrm{Na}+$, $\mathrm{K}(+)$ - ATPase, as a mechanism of $\mathrm{Cl}$ - uptake [71], as pathways for endocytosis of neural cell adhesion molecules (NCAM) [72], and for receptor-mediated vesicular transport [73], such as internalization of metabotropic glutamate receptors [74].

\section{Plasma Membrane Damage in Human Severe Traumatic Brain Oedema}

In patients with brain trauma and moderate oedema, some non-pyramidal neurons show a well preserved and continuous plasma membrane, swollen mitochondria [75,76] (Figure 5). In severe oedema of traumatic brain injuries complicated with subdural hematoma, the non-pyramidal neurons, astrocytes and oligodendrocytes show plasma membrane fragmentation, swollen mitochondria, enlargement of rough and smooth endoplasmic reticulum cisterns, and irregular dilation of nuclear envelope [77]. The rough endoplasmic reticulum displays extensive areas with detachment of membrane-bound associated ribosomes, and a marked reduction in the number of polysomes [78] (Figure 6).

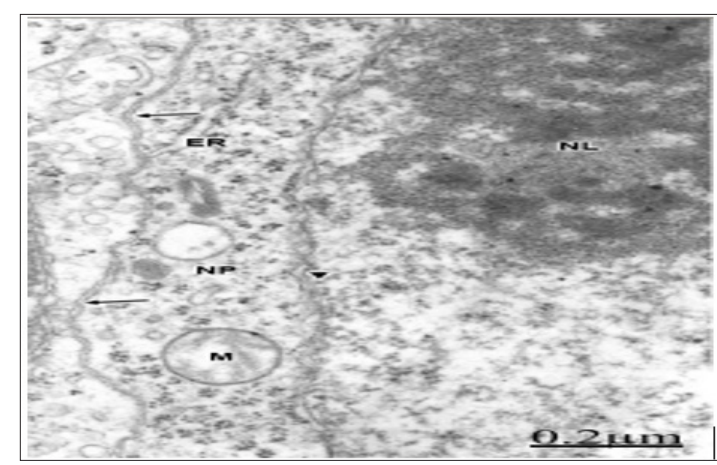

Figure 5: Brain trauma. Left frontal hematoma. Non-pyramidal neuron in an area of moderate perifocal oedema bearing a continuous plasma membrane (arrows), a non-dilated endoplasmic reticulum cistern (ER) and nuclear envelope (arrowhead), and swollen mitochondria (M). Note the well-preserved nucleolar substructures (NL).X 30.000 .

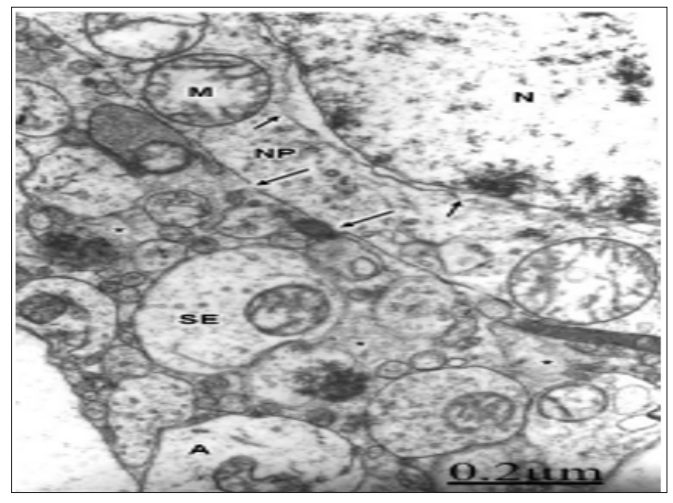

Figure 6: Brain trauma. Subdural hematoma. Left parietal cortex. Severe oedema. Non-pyramidal neuron (NP) displaying disrupted plasma membrane (long arrows), irregularly dilated nuclear envelope (short arrows), and swollen mitochondria $(\mathrm{M})$. In the neighbouring neuropil, the asterisks label the enlarged extracellular space containing proteinaceous oedema fluid, which separates degenerated synaptic endings (SE), and swollen astrocytic processes (A). Note the swollen nucleoplasm (N). X. 30.000.

Singleton and Povlishock, Farkas demonstrated disruption of neuronal somatic plasmalemma in diffuse traumatic brain injury. Areas of focal necrosis of plasma membrane, cytomembranes, mainly outer nuclear membrane, smooth membranes of Golgi endoplasmic sacs, and lysosomes are observed [79] (Figure 7). In severe traumatic brain injuries with enlarged extracellular spaces containing proteinaceous oedema fluid, the plasma membrane of neurons appears. The degenerated myelinated axons show invaginations of the axolemmal membrane and formation of endocytic vesicles $\mathrm{x}$. The myelin membranes appear separated forming large intra-period vacuoles. Traumatic damage of axolemmal membrane was reported by Castejon, Genarelli, Erb, Povlishock, and Maxswell.

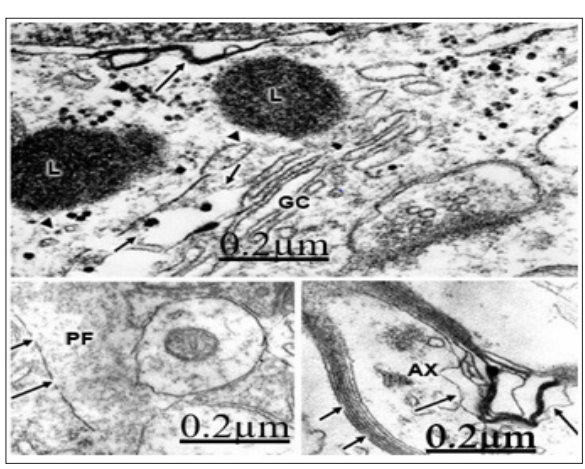

Figure 7: Brain trauma. Subdural hematoma. Right parietal cortex. Non-pyramidal neuron showing focal necrosis of nuclear envelope (long arrow) and of smooth Golgi complex (GC) membranes (short arrows). The lysosomes (L) show a discontinuous globular limiting membrane (arrowheads). X 75.000 .

\section{The Damage of Astrocyte Membranes}

The swollen and clear astrocytes display marked degenerative changes induced by brain edema in comparison with those 
exhibited by neuronal cells. Areas of focal necrosis and fragmented limiting plasma membrane, over-distended rough endoplasmic reticulum cisterns with extended degranulated areas, and vacuoles of smooth endoplasmic reticulum with necrotic limiting membrane are found [80-82] (Figure 8). Oligodendroglial cells show also marked edematous changes featured by lacunar enlargement of rough endoplasmic reticulum and nuclear envelope, detachment of membrane bound ribosomes, swollen mitochondria associated to rough endoplasmic reticulum, and discontinuous plasma membrane [83,84]. (Figure 9). The endoplasmic reticulum is a subcellular compartment playing a fundamental role in the folding and processing of newly synthesized membranes and secretory processes, reactions which are strictly calcium/dependent. Severe disruption of neuronal $\mathrm{Ca} 2+$ homeostasis leading to a lethal $\mathrm{Ca} 2+$ overload as occurs in brain ischemia, can initiate a cascade of destructive processes which lead to the death of neurons during cerebral ischemia [85-86]. As stated above endoplasmic reticulum function is disturbed in many acute and chronic diseases of the brain, such as Parkinson's and Alzheimer's diseases [87]. Therefore, endoplasmic reticulum dysfunction is basically important to elucidate the pathogenetic mechanisms of neurodegeneration.

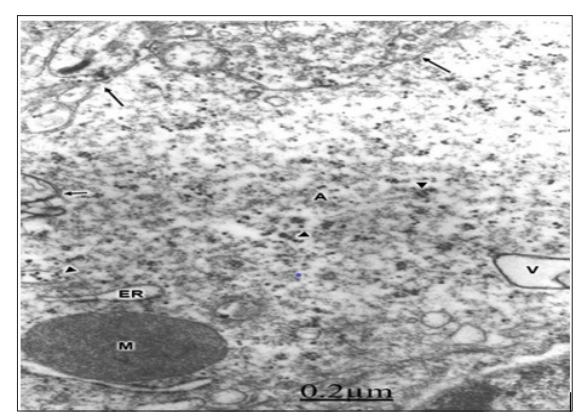

Figure 8: Brain trauma. Swollen and clear astrocyte cell (A) depicting fragmented plasma membrane (long arrows) and areas of focal necrosis (short arrow), degranulated and dilated rough endoplasmic reticulum cisterns (ER), glycogen granules (arrowheads), and a vacuole (V) limited by necrotic membrane. Note the degenerated and dense mitochondrion $(\mathrm{M})$ associated with rough endoplasmic reticulum X. 60.000 .

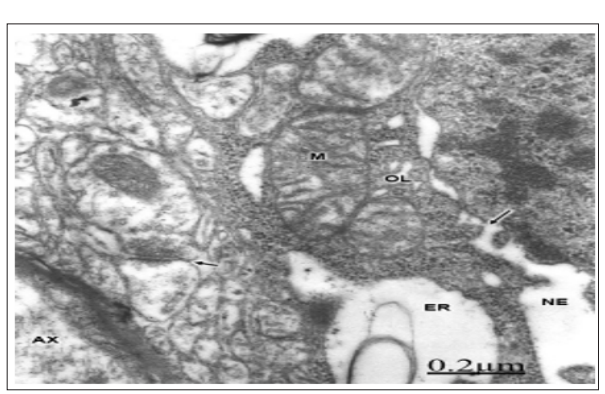

Figure 9: Brain trauma. Hydropic oligodendrocyte (OL) showing over-distended perinuclear $(\mathrm{PN})$ and endoplasmic reticulum (ER) cisterns, disassembly of nuclear envelope (NE), and nuclear pores (long arrow). Note the swollen mitochondria (M) associated to rough endoplasmic reticulum. The neighboring neuropile exhibits degenerated axodendritic synapse (short arrow) and myelinated axon (AX). X 60.000 .
Peroxidative stress has been implicated in mechanism leading to neuronal cell injury [88-91]. One source of free radicals in ischemic cells is arachidonic acid released by membrane phospholipids under the action of Ca2+ activated phospholipase A2 [92]. Several oxygen radical species besides superoxide radicals are produced following hypoxia. Superoxide radicals have been shown to change phospholipid and protein structure. Hydroxyl radicals are the most reactive and are known to initiate lipid peroxidation and protein oxidation $[93,94]$.

\section{Brain Trauma and Synaptic Membrane Specialized Contact Alterations}

In congenital hydrocephalus and in severe oedematous regions of complicated traumatic brain injuries, synaptic plasticity, synaptic degeneration and occur. Synaptic disassembly featured by wide separation of pre- and postsynaptic membranes and loss of peri synaptic astrocytic glial escheatment also is observed [95,96] (Figure 10).

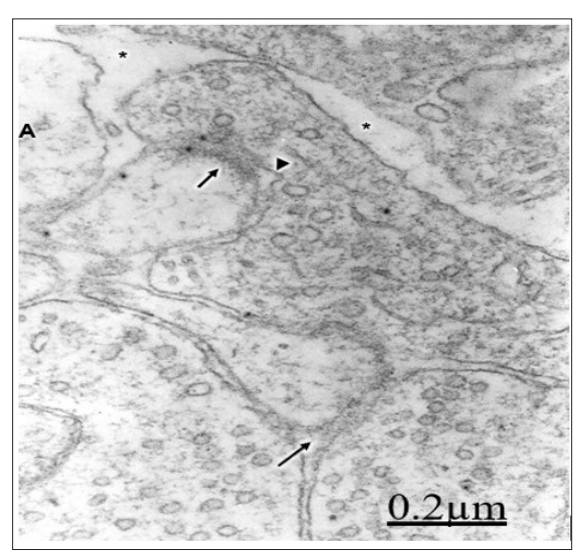

Figure 10: Brain trauma. Subdural hematoma. Left parietal cortex. Severely edematous neuropil showing synaptic disassembly and disappearance of pre- and postsynaptic densities (long arrow) of an axospinodendritic contact. The short arrow labels another spine synapse exhibiting partial vestiges of the postsynaptic density, and irregular profile of presynaptic membrane (arrowhead). Note the expansion of extracellular space (asterisks), and the detachment of peri synaptic astrocytic cytoplasm (A). X. 60.000 .

\section{Synaptic Plasticity on Severe Human Traumatic Injuries}

Numerous flat, curved and invaginated axodendritic and axospinous asymmetric synapses, and a less proportion of axodendritic and axosomatic symmetric synapses are found. Activated or sensitized synapses showed numerous frontline spheroid synaptic vesicles, prominent dense presynaptic dense projections and increased in length of synaptic membrane complex. Perforated synapses, multiple synapses and serial synapses are also found evincing synaptic splitting and formation of new synaptic connections (Figure 11).

\section{Synaptic Degeneration on Severe Human Traumatic Injuries}

The hydrocephalic or interstitial clear edema, the hematogenous edema fluid in traumatic brain injuries, and the proteinaceous 
edema fluid in brain tumors accumulated in the dilated extracellular space of cerebral cortex neuropil induce swelling and shrinkage of pre- and postsynaptic structures, increased amount of presynaptic axoplasmic granular substance, and clumping, enlargement and depletion of synaptic vesicles, and filamentous hypertrophy of presynaptic endings (Figure 12). Disappearance of synaptic densities is evident in some cases. In severe brain edema, swollen and shrunken presynaptic endings with discontinuous limiting plasma appear separated from the postsynaptic structures and detached from the glial ensheathment expressing synaptic disassembly. Phagocytosis of isolated presynaptic endings, and of the entire synaptic contacts by astrocytes, microglial cells, and by non-nervous invading cells, such as monocytes and macrophages, are frequently observed [97].

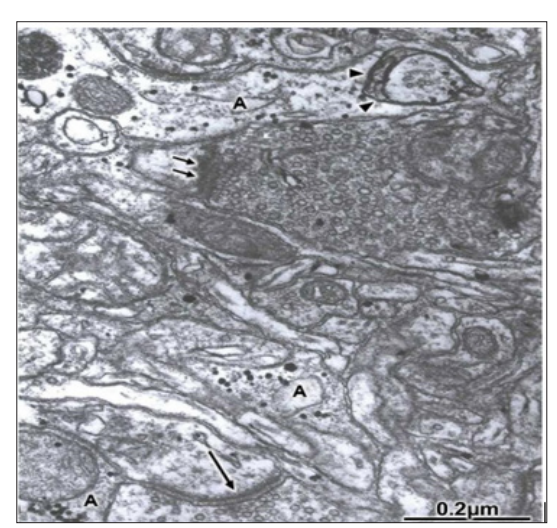

Figure 11: Brain trauma. Epidural hematoma. Right temporal cortex. Moderate edematous region. An asymmetric ax spinous synapse (long arrow) appears showing a curved arrangement of the synaptic membrane complex. Another activated and flat symmetrical synapse (short arrows) is observed at the upper part of the figure. Note the peri synaptic glycogen-rich-astrocytic ensheathment (A) phagocyting a degenerated presynaptic ending (arrowheads).

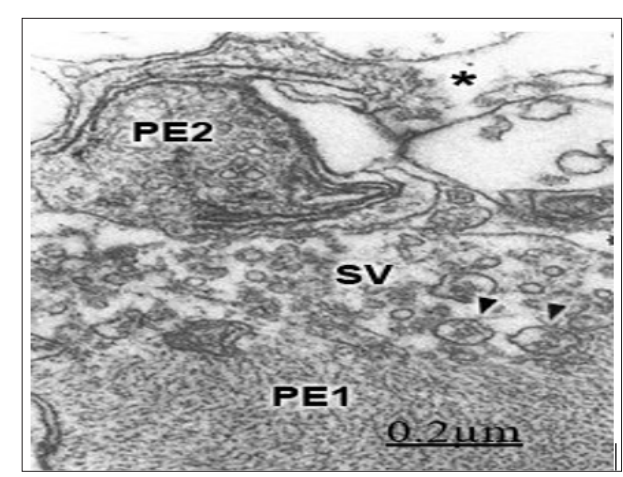

Figure 12: Brain trauma. Right parietal cortex. Subdural hematoma. Degenerated presynaptic ending (PE1) exhibiting filamentous hypertrophy and reduced number of displaced synaptic vesicles (SV). Large vesicles (arrowheads) containing degenerated and small dense-cored nuclei are also observed. Another severely degenerated presynaptic ending (PE2) shows necrotic osmiophilic membranes, dense axoplasmic substance and focal necrotic areas. The asterisk labels the enlarged extracellular space containing non-proteinaceous edema fluid.

\section{The Damage of Interastrocytary Gap Junctions}

Disruption and disassembly of interastrocytary gap junctions have been observed by us in human edematous cerebral cortex of patients with traumatic brain injuries and brain tumors [98] (Figure 13).

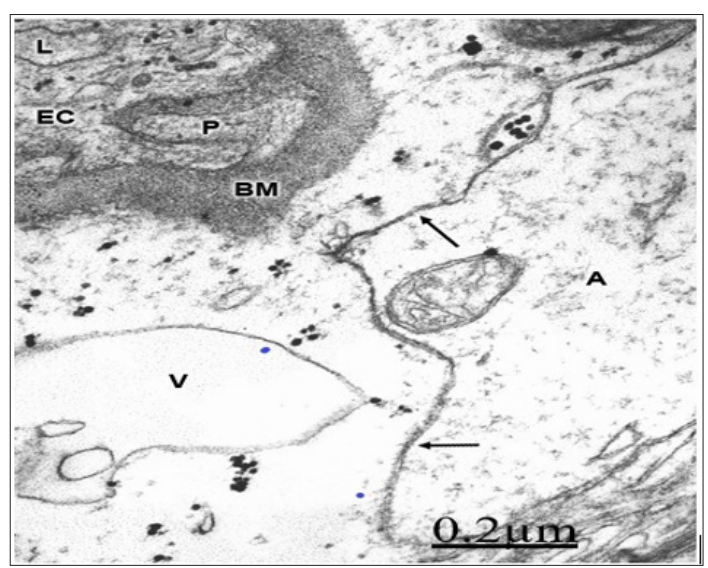

Figure 13: Anomaly of anterior cerebral artery. Left parietal cortex. Astrocytic end-feet (A) showing extensive and undulated interastrocytary gap junction and membrane domains with fusion of both confronted membranes (long arrows). Note the vacuolization (V) and swelling of perivascular astrocytic end feet, and the dense capillary basement membrane $(\mathrm{BM})$. The pericyte cytoplasm $(\mathrm{P})$, the endothelial cell (EC), and the capillary lumen (L) are also distinguished. X 36.000 .

In severe traumatic brain injuries, gap junction disruption and disassembly are observed. Gap junction disassembly is characterized by wide separation of astrocytic end- feet confronted membranes [98] (Figure 14)

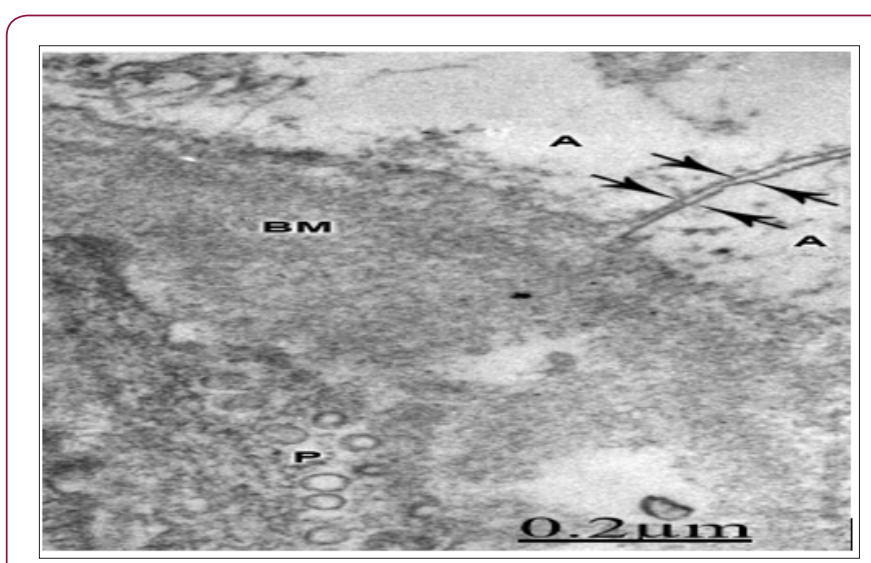

Figure 14: Brain trauma. Right epidural hematoma. Right temporal cortex. Capillary (C) showing the increased activity of endothelial cell luminal membrane, which exhibits the formation of pinocytotic vesicle (short arrow), deep invagination (long arrow), and formation of endothelial vacuoles $(\mathrm{V})$. Note the swollen basement membrane (BM), and the degenerated myelinated axons (AX) in the neighboring neuropil. The arrow indicates the disrupted axolemmal membrane, and the circle the granular degeneration of neurofilaments. X 36.000 . 
According to Hossain [99] rat astrocytes respond to ischemic insult by reorganizing their gap junction and increasing the junctional protein connexin43. Ochalski [100] reported extensive gap junctions, disruption and gap junction disassembly in kainic lesion sites of rat brain. Theriault described gap junction remodeling in rat spinal cord after acute compression injury. Li [101] found astrocytic gap junction internalization in rat brain after cerebral focal ischemia. Figure 15 Soroceanu [102] reported that highgrade brain tumors show reduced intercellular communication, and a decrease in connexin-43 protein levels. Aronica [103] found high expression of connexin proteins in low grade tumors, and in the peritumoral reactive astrocytes. Nakase [104-110] reported a protective role of astrocytic gap junctions in ischemic stroke reducing apoptosis and inflammation following ischemic insult. According to Perez- Velazquez [106,111-115], the actions of gap junctional coupling during injuries may be causally related to oxidative stress.

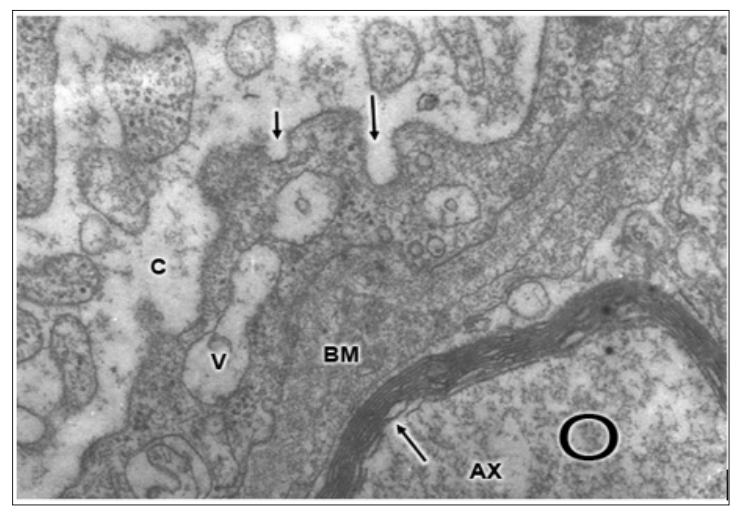

Figure 15: Brain trauma. Subdural parieto-temporal hematoma. Left parietal cortex. Disassembly of interastrocytary gap junctions featured by wide separation of confronted astrocytic end feet membrane (A). Note the swollen and vacuolated basement membrane (BM), and the increased vesicular transport in the pericytal cytoplasm (P). X 90.000.

\section{The Activity of Endothelial Cell Luminal Membrane of Cerebral Capillaries}

In severe and complicated traumatic brain injuries, the endothelial cell luminal membrane of brain capillaries undergoes profound activity changes that characterize the increased cerebrovascular permeability. Such changes are increased formation of micro- and macropinocytotic vesicles and clathrinidcoated vesicles, deep invaginations and formation of incomplete trans endothelia channels, and emission of pseudopods to form endothelial vacuoles [107,115-118].

\section{Concluding Remarks}

In moderate brain oedema a continuous plasma membrane is observed in some neurons, but the cytoplasmic membranes, such as smooth and rough endoplasmic reticulum membranes appear damaged. In severe oedema, fragmentation of plasma membrane, enlargement and focal necrosis of rough endoplasmic cisterns and nuclear envelope, detachment of membrane-bound ribosomes, and reduction of polysome are found [118]. Shallow and deep invaginations of plasma membrane, and the formation of endocytic and clathrin-coated vesicles are seen. In astrocyte cells, areas of focal necrosis and fragmented limiting plasma membrane, overdistended rough endoplasmic reticulum cisterns with extense degranulated membrane domains, and vacuoles of smooth endoplasmic reticulum with necrotic limiting membrane are observed. Oligodendroglia cells show also notably edematous changes featured by lacunar enlargement of rough endoplasmic reticulum and nuclear envelope, detachment of membrane bound ribosomes, and discontinuous plasma membrane [119-121]. Plastic changes and damage of synaptic membranes are found. Synaptic vesicle exocytosis at the synaptic active zone, and endocytosis at the non-specialized regions of presynaptic ending limiting membrane are frequently observed at activated synapses.

In severe brain edema, synaptic disassembly occurs featured by wide separation of pre- and postsynaptic membranes and loss of peri synaptic astrocytic glial escheatment. Disruption, fusion and disassembly of interastrocytary gap junctions have also been observed. The endothelial cell luminal membrane of brain capillaries undergoes profound activity changes that characterize increased cerebrovascular permeability, such as increased formation of micro- and macropinocytotic vesicles, clathrin coated vesicles, and emission of pseudopods to form endothelial vacuoles, The alterations of nerve cell plasma membranes and cytomembranes are related with the anoxic-ischemic conditions of brain parenchyma [121-124]. The role of free radical and lipid peroxidation, disturbed energy metabolism, altered metabolic cascades, glutamate excitotoxicity, haemoglobin toxicity, protein aggregation, and presence of extracellular oedema fluid are discussed in relation with the derangement of nerve cells membranes.

\section{Acknowledgement}

This paper was carried out through the logistic support of Biological Research Institute. Faculty of Medicine. Zulia University and Castejon Foundation.

\section{References}

1. Bass NH, Hess HH, Pope A (1974) Altered cell membranes in CreutzfeldtJakob disease. Microchemical studies Arch Neurol 31(3): 174-182.

2. Brown FR, Chen WW, Kirschner DA, Frayer KL, Powers JM, et al. (1983) Myelin membrane from adrenoleukodystrophy brain white matterhistochemical properties. J Neurochem 41(2): 341-348.

3. Malunova LB, Samoilov MO (1984) Early postanoxic changes in the neuronal and neuropil ultrastructure of the cerebral cortex in cats. Arkh Anat Gistol Embriol 86(1): 46-49.

4. Cohadon F (1984) Cell membrane alterations during situations of acute stress to the cerebral parenchyma. Mechanisms, consequences and therapeutic perspectives. Neurochirurgie 30: 69-83.

5. Coria F, Silos I, Fernandez R, Monton F, Lafarga M (1985) Demyelinationinduced plasticity in the axon membrane: an ultrastructural cytochemical study of lead neuropathy in the rat. Neurosci Lett 58: 359-364.

6. Roos RA, Bots GT, Hermans J (1985) Neuronal nuclear membrane indentation and astrocyte/neuron ratio in Huntington's disease. A quantitative electron microscopic study J Hirnforsch 26(6): 689-693. 
7. Salvati S, D’Urso D, Conti Devirgiliis L, Serlupi Crescenzi G (1986) Biochemical changes in central nervous system membranes in experimental allergic encephalomyelitis. J Neurochem 47(1): 239-244.

8. Black JA, Waxman SG, Smith ME (1987) Macromolecular structure of axonal membrane during acute experimental allergic encephalomyelitis in rat in guinea pig spinal cord. J Neuropathol Exp Neurol 46(2): 167184.

9. Waxman SG, Black JA, Duncan ID, Ransom BR (1990) Macromolecular structure of axon membrane and action potential conduction in melin deficient and myelin deficient heterozygote rat optic nerves. J Neurocytol 19(1): 11-28

10. Cuevas P, Gutierrez Díaz JA, Dujovny M, Diaz FG, Ausman JI (1988) Freeze-fracture cytochemistry of cholesterol content in neuronal plasma membrane following cerebral ischemia. Neurol Res 10(1): 2-6.

11. Pico RM, Gall C (1989) Continuities between outer nuclear membrane and the rough endoplasmic reticulum increase in hippocampal neurons during seizure-induced protein synthesis. Brain Res 497(2): 387-392.

12. Saatman KE, Bozyczko Coyne D, Marcy V, Siman R, McIntosh TK (1996) Prolonged calpain-mediated spectra in breakdown occurs regionally following experimental brain injury in the rat. J Neuropathol Exp Neurol 55(7): 850-860.

13. Homayoun P, Rodríguez De Turco EB, Parking NE, Lance DC, Soblosky J (1997) Delayed phospholipids degradation in rat brain after traumatic brain injury. J Neurochem 69(1): 199-205.

14. Paschen W, Doutheil J (1999) Disturbance of endoplasmic reticulum functions: A key mechanism underlying cell damage? In Baethmann A, Glesnila N, Ringel F, Eriskat, J (Eds.). Current Progress in the Understanding of Secondary Brain Damage from Trauma and Ischemia. Acta Neurochirug. (Wien): (Suppl.) 73; Springer p. 1-5.

15. Hu BR, Janelidze S, Ginsberg MD, Busto R, Perez Pinzon M (2001) Protein aggregation after focal brain ischemia and reperfusion. J Cereb Blood Flow Metab 21(7): 865-875.

16. Mengesdorf T Proud CG, Mies G, Paschen W (2002) Mechanisms underlying suppression of protein synthesis induced by transient focal cerebral ischemia in mouse brain. Exp Neurol 177(2): 538-546.

17. Gennarelli TA, Tipperman R, Maxwell WL, Graham DI, Adams JH, et al. (1993) Traumatic damage to the nodal axolemma: an early, secondary injury. Acta Neurochir 57: 49-52.

18. Castejón OJ (1985) Electron microscopic study of central axonal degeneration in traumatic human brain edema. J Submicroscopic Cytol 17(4): 703-718.

19. Castejón OJ, Acurero G (2005) Traumatic axolemmal and cytoskeletal derangement and loss of consciousness. J Submicrosc Cytol Pathol 36 285-293.

20. Homayoun P, Rodríguez De Turco EB, Parking NE, Lance DC, Soblosky J, et al. (1997) Delayed phospholipids degradation in rat brain after traumatic brain injury. J Neurochem 69(1): 199-205.

21. Babu PP, Kumari LR, Vemuri MC (1994) Differential changes in cell morphology, macromolecular composition and membrane protein profiles of neurons and astrocytes in chronic ethanol treated rats. Mol Cell Biochem 130(1): 29-40.

22. Dux E, Oschlies U, Uto A, Kusumoto M, Hossman KA (1996) Early ultrastructural changes after brief histotoxic hypoxia in cultured cortical and hippocampal CA1 neurons. Acta Neuropathol 92(6): 541-544.

23. Maxwell WL, Kosanlavit R, McCreath BJ, Reid O, Graham DI (1999) Freeze-fracture and cytochemical evidence for structural and functional alteration in the axolemma and myelin sheath of adult guinea pig optic nerve fibers after stretch injury. J Neurotrauma 16(4): 273-284.

24. Paschen W (1996) Glutamate excitotoxicity in transient global cerebral ischemia. Acta Neurobiol Exp (Wars) 56(1): 313-322.
25. Dabrowskam Bouta B, Sulkowski G, Bartosz G, Walski M, Rafalowska U (1999) Chronic lead intoxication affects the myelin membrane status in the central nervous system of adult rats. J Mol Neurosci. 13(1): 127-139.

26. Dabrowska Bouta B, Sulkowski G, Walski M, Struzynska L, Lenkiewicz, et al. (2000) Acute lead intoxication in vivo affects myelin membrane morphology and CNP ase activity. Exp Toxicol Pathol 52(3): 257-263.

27. Torp R, Head E, Milgram NW, Hahn F, Otersen OP, et al. (2000) Ultrastructural evidence of fibrillar beta-amyloid associated with neuronal membranes in behaviorally characterized aged dog brains. Neuroscience, 96(3): 495-506.

28. Yamaguchi H, Maat Schieman ML, van Duinen SG, Prins FA, Neeskens P (2000) Amyloid beta protein (Abeta) starts to deposit as plasma membrane-bound form in di fuse plaques of brains from hereditary cerebral hemorrhage with amyloidosis-Dutch type, Alzheimer disease and nondemented aged subjects. J Neuropathol Exp Neurol 59(8): 723732 .

29. Paschen W (1996) Glutamate excitotoxicity in transient global cerebral ischemia. Acta Neurobiol Exp (Wars) 56(1): 313-322.

30. Castejón OJ, Díaz M, Valero C (1997) Light and electron microscopy of nerve cells in traumatic oedematous human cerebral cortex. Brain Inj 11(5): 363-388.

31. Ginsberg MD, Watson BD, Busto R (1988) Peroxidative damage to cell membranes following cerebral ischemia A cause of ischemia brain injury? Neurochem Pathol 9: 171-193.

32. Uriu T Omori K, Yamamoto A, Inoue M, Inagaki C, et al. (1991) Two types of clathrin-coated vesicles an isolated from rat brain: analysis, biochemical properties and cellular origin. J Neurochem 56: 1548-1556.

33. Choi BH (1993) Oxygen, antioxidants and brain dysfunction. Yonsei Med J 34(1): 1-10.

34. Evans PH (1993) Free radicals in brain metabolism and pathology. $\mathrm{Br}$ Med Bull J 49(3): 577-587.

35. Boldyrev A, Song R, Dyatlov VA, Lawrence DA, Carpenter DO (2000) Neuronal cell death and reactive oxygen species. Cell Mol Neurobiol 20(4): 433-450

36. Paschen W, Doutheil J (1999) Disturbance of endoplasmic reticulum functions: A key mechanism underlying cell damage? In Baethmann A, Glesnila N, Ringel F, Eriskat J (Eds.) Current Progress in the Understanding of Secondary Brain Damage from Trauma and Ischemia. Acta Neurochirug. (Wien) p. 1-5.

37. Paschen W, Mengesdorf T, Althausen S, Hotop S (2001) Peroxidative stress selectively down-regulates the neuronal stress response activated under conditions of endoplasmic reticulum dysfunction. J Neurochem 76: 1916-1924.

38. Paschen W (2003) Endoplasmic reticulum: a primary target in various acute disorders and degenerative diseases of the brain. Cell Calcium 34(4-5): 365-383.

39. Doutheil J, Althausen S, Treiman M, Paschen W (2000) Effect of nitric oxide on endoplasmic reticulum calcium homeostasis, protein synthesis and energy metabolism. Cell Calcium 27(2): 107-115.

40. Castejón 0J, Arismendi GJ (2003) Morphological changes of dendrites in the human edematous cerebral cortex, A transmission electron microscopic study. J Submicrosc Cytol Pathol 35: 395-413

41. Castejón OJ, Castejón HV (2004) Structural patterns of injured mitochondria inhuman oedematous cerebral cortex. Brain Injury 18(11): 1107-1126.

42. Castejón OJ (2004b) Lysosome abnormalities and lipofucsin content of nerve cells of edematous cerebral cortex. J Submicrosc Cytol Pathol 36(3-4): 263-271.

43. Castejón, OJ. Castellano A, Arismendi GJ, Medina Z (2005) The 
inflammatory reaction inhuman traumatic brain edema. J Submicrosc Cytol Pathol 37(1): 43-52.

44. Sponne I, Fifre A, Koziel V, Oster T, Olivier JL, et al. (2004) Membrane cholesterol interferes with neuronal apoptosis induced by soluble oligomers but not fibrils of amyloid-bet peptide. Faseb J 18(7): 836-838.

45. Verdier Y, Zarandi M, Penke B (2004) Amyloid bet-peptide interactions with neuronal interactions with neuronal and glial cell plasma membrane: binding sites and implications for Alzheimer's disease. J Pept Sci 10(5): 229-248.

46. Hayashi T, Abe K (2004) Ischemic neuronal cell death and organellae damage. Neurol Res 26(8): 827-834.

47. Singleton RH, Povlishock JT (2004) Identification and characterization of heterogeneous neuronal injury and death in regions of diffuse brain injury: evidence for multiple independent injury phenotypes. J Neurosci 24(1): 3543-3553.

48. Luo J, Shi R(2004) Acrolein induces axolemmal disruption, oxidative stress, and mitochondrial impairment in spinal cord tissue. Neurochem Int 44(7): 475-486.

49. Kurnellas MP, Nicot A, Shull GE, Elkabes S (2005) Plasma membrane calcium ATPase deficiency causes neuronal pathology in the spinal cord: A potential mechanism for neurodegeneration in multiple sclerosis and spinal cord injury. FASEB J 19(2): 298-300.

50. Farkas O, Lifschitz J, Povlishock JT (2006) Mechanoporation induced by diffuse traumatic brain injury: an irreversible or reversible response to injury? J Neurosci 26(12): 3130-3140.

51. Thompson RJ, Zhou N, MacVicar BA (2006) Ischemia opens neuronal gap junction hemichannels. Science 312(5775): 924-927.

52. Calabrese V, Boyd-Kimball D, Scapagnini G, Butterfield DA (2004) Nitric oxide and cellular stress response in brain aging and neurodegenerative disorders: The role of vitagenes. In Vivo 18(3): 245-267.

53. Wang X, Wang F, Arterburn L, Wollmann R, Ma J (2006) The interaction between cytoplasmic prion protein and the hydrophobic lipid core of membrane correlates with neurotoxicity. J Biol Chem 281(19):1355613559.

54. Arduíno DM, Esteves AR, Cardoso SM and Oliveira CR (2009) Endoplasmic reticulum and mitochondria interplay mediates apoptotic cell death: relevance to Parkinson's disease. Neurochem Int 55(5): 341348.

55. Lin W, Popko B (2009) Endoplasmic reticulum stress in disorders of myelinating cells. Nat Neurosci 12(4): 379-85.

56. Walker AK, Atkin JD (2011) Stress signaling from the endoplasmic reticulum: A central player in the pathogenesis of amyotrophic lateral sclerosis. IUBMB Life 63(9): 754-63.

57. Bellucci A, Zaltieri M, Navarria L, Grigoletto J, Missale C, Spano P (2012) From $\alpha$-synuclein to synaptic dysfunctions: New insights into the pathophysiology of Parkinson's disease. Brain Res 1476: 183-202.

58. Hedskog L, Pinho CM, Filadi R, Rönnbäck A, Hertwig L, Wiehager B, et al. (2013) Modulation of the endoplasmic reticulum-mitochondria interface in Alzheimer's disease and related models. Proc Natl Acad Sci U S A 110(19): 7916-7921.

59. Calvo Rodríguez M, García-Durillo M, Villalobos C, Núñez L (2016) In vitro aging promotes endoplasmic reticulum (ER)-mitochondria $\mathrm{Ca}^{+}$cross talk and loss of store-operated Ca2+ entry (SOCE) in rat hippocampal neurons. Biochim Biophys Acta 1863(11): 2637-2649.

60. Paillusson S, Gomez Suaga P, Stoica R, Little D, Gissen P, Devine MJ, et al. (2017) $\alpha$-Synuclein binds to the ER-mitochondria tethering protein VAPB to disrupt $\mathrm{Ca}^{+}$homeostasis and mitochondrial ATP production. Acta Neuropathol 134(1): 129-149.

61. Szydlowska K, Tymianski M (2010) Calcium, ischemia and excitotoxicity. Cell Calcium. 47(2): 122-129.
62. Ghavami S, Shojaei S, Yeganeh B, Ande SR, Jangamreddy JR, et al. (2014) Autophagy and apoptosis dysfunctionin neurodegenerative disorders. Prog Neurobiol 112: 24-49.

63. Castejón Orlando J (2015) Ultrastructural pathology of oligodendroglial cells in traumatic and hydrocephalic human brain Edema: A Review. Ultrastructural Pathol 39(6): 359-368.

64. Castejon Orlando J (2017) Synaptic plasticity in severe human traumatic brain injuries: An electron microscopic study using cortical biopsies. J Adv Microsc Res 12(2): 97-103.

65. Castejon Orlando J (2017) The blood brain barrier breakdown in severe traumatic brain injuries in aging patients. An Electron Microscopic Study. J Adv Microsc Res 12(3): 236-240.

66. Castejon Orlando J (2018) Ultrastructural pathology of Golgi apparatus of nerve cells in human brain edema. Biomed J Sci \& Tech Res DOI:10.26717/BJSTR.2018.08.001679.

67. Castejón Orlando J (1994) Transmission electron microscopic study of human hydrocephalic cerebral cortex. J Submicrosc Cytol Pathol 26(1): 29-39.

68. Castejón Orlando J, Díaz M, Valero C (1994) Ultrastructural alterations of Golgi apparatus in the nerve cells of cerebral cortex in human hydrocephalus. A qualitative study using cortical biopsies. Scanning Microscopy 8(1): 89-96.

69. Surchev L, Dontchev V, Ichev K, Dolapchieva S., Boshilova-Pastirova A, et al. (1995) Changes in the neuronal plasma membrane during synaptogenesis. Cell Mol Biol 41(8): 1073-1080.

70. Blanpied TA, Scott DB, Ehlers MD (2002) Dynamic and regulation of clathrin coats as specialized endocytic zones of dendrites and spines. Neuron 39(6): 435-439.

71. Uriu T, Omori K, Omori K, Yamamoto A, Inoue M, et al. (1991) Two types of clathrin-coated vesicles an isolated from rat brain: analysis, biochemical properties and cellular origin. J Neurochem 56(5): 15481556.

72. Minana R, Duran JM, Tomas M, Renau-Piqueras J, Guerri C (2001) Neural cell adhesion molecule is endocytosed via clathrin-dependent pathway. Eur J Neurosci 13(4): 749-756.

73. Pley U, Parham P (1993) Clathrin: Its role in receptor-mediated vesicular transport and specialized functions in neurons. Crit Rev Biochem Mol Biol 28(5): 431-464.

74. Albasanz JL, León D, Ruíz MA, Fernández M, Martín M (2002) Adenosine A1 receptor agonist treatment up-regulates rat brain metabotropic glutamate receptors. Biochim Biophys Acta 1593(1): 69-75.

75. Castejón OJ, Castejón HV, Castellano A (2001) Oligodendroglial cell damage and demyelination in infant hydrocephalus. An electron microscopy study. J Submicrosc Cytol Pathol 33(1-2): 33-40.

76. Castejón OJ, Díaz M, Valero C (1997) Light and electron microscopy of nerve cells in traumatic oedematous human cerebral cortex. Brain Inj 11(5): 363-388.

77. Castejón OJ (2004) Structural pattern of injured mitochondria in oedematous human cerebellar cortex. Brain Injury 18(11): 1107-1126.

78. Castejón OJ, Castejón HV, Diaz M, Castellano A (2001) Consecutive light microscopy, scanning-transmission electron microscopy and transmission electron microscopy of traumatic human brain oedema and ischaemic brain damage. Histol Histopathol 16(1): 1117-1134.

79. Castejón OJ, Díaz M, Valero C (1994) Ultrastructural alterations of Golgi apparatus in the nerve cells of cerebral cortex in human hydrocephalus. A qualitative study using cortical biopsies Scanning Microscopy 8(1): 89-96.

80. Castejón OJ (1998) Morphological astrocytic changes in complicated human brain trauma. A light and electron microscopic study. Brain Inj 12(5): 409-427. 
81. Castejón OJ, Díaz M, Castejón HV, Castellano A (2002) Glycogen-rich and glycogen-depleted astrocytes in the oedematous human cerebral cortex associated with brain trauma, tumours and congenital malformations an electron microscopy study. Brain Injury 16(2): 109-132.

82. Castejón OJ (2013) Electron microscopy of astrocyte changes and subtypes in traumatic human edematous cerebral cortex: a review. Ultrastruct Pathol 37(6): 417-424.

83. Castejón OJ, Castejón HV (2000) Oligodendroglial cell behaviour in traumatic oedematous human cerebral cortex. A light and electron microscopic study. Brain Injury 14(4): 303-317.

84. Castejón OJ (2011) Correlative microscopy of cerebellar neuroglial cells. J. Adv. Microsc. Res. 6(3): 159-176.

85. Morley P, Tauskela JS, Hakim AM (1999) Calcium Overload. Cerebral Ischemia New Jersey: Humana Press p. 69-104.

86. Paschen W (2000) Role of calcium in neuronal cell injury: which subcellular compartment is involved? Brain Res Bull 53(4): 409- 413.

87. Paschen W, Frandsen A (2001) Endoplasmic reticulum dysfunction--a common denominator for cell injury in acute and degenerative diseases of the brain? J Neurochem 79(4): 719-725.

88. Evans PH (1993) Free radicals in brain metabolism and pathology. Br Med Bull 49(3): 577-587.

89. Choi BH (1993) Oxygen, antioxidants and brain dysfunction. Yonsei Med J 34(1): 1-10

90. Paschen W, Mengesdorf T, Althausen S, Hotop S (2001) Peroxidative stress selectively down-regulates the neuronal stress response activated under conditions of endoplasmic reticulum dysfunction. J. Neurochem 76(6): 1916-1924.

91. Boldyrev A, Song R, Dyatlov VA, Lawrence DA, Carpenter DO (2000). Neuronal cell death and reactive oxygen species. Cell Mol Neurobiol 20(4): 433-450.

92. Keuhl FA, Egans RW (1980) Prostaglandins, arachidonic acid and inflammation. Science 210(4473): 978-984.

93. Ginsberg MD, Watson BD, Busto R, Yoshida S, Prado R, et al. (1988) Peroxidative damage to cell membranes following cerebral ischemia. A cause of ischemia brain injury? Neurochem Pathol 9: 171-193.

94. Siesjo BK, Aquardh CD, Bengtson F (1989) Free radicals and brain damage. Cerebrovasc Brain Metab Rev 1(3): 165-211.

95. Castejón OJ (2003) Synaptic plasticity in the oedematous human cerebral cortex. J Submicrosc Cytol Pathol 35(2): 177-197.

96. Castejón OJ (2015) Synaptic plasticity and synaptic degeneration in human congenital hydrocephalus. J Pedriat Neurol 6(2): 99-107.

97. Castejón OJ, Díaz M, Valero C (1995) Synaptic degenerative changes in human traumatic brain edema. J Neurosurg Sci 39(1): 47-65.

98. Castejón OJ (1980) Electron microscopy study of capillary wall in human cerebral edema. J Neuropathol Exp Neurol 39(3): 296-328.

99. Hossain MZ, Peeling J, Sutherland R, Hertzberg EL, Nagy JI (1994) Ischemia-induced cellular redistribution of the astrocytic gap junctional protein connexin43 in rat brain. Brain Res 652(2): 311-322.

100. Ochalski PA, Sawchuk MA, Hertzberg E, and Nagy JI (1995) Astrocytic gap junction removal, connexin3 redistribution, and epitope masking at excitatory amino acid lesion sites in rat brain. Glia 14(4): 279-294.

101. Li WE, Ochalski PA, Hertzberg EL, Nagy JI (1998) Immunorecognition, ultrastructure and phosphylatin status of astrocytic gap junctions and connexin43 in rat brain after cerebral focal ischaemia. Eur J Neurosci 10(7): 2444-2463.

102. Soroceanu L, Manning TJ, Sontheimer H (2001) Reduced expressionof conexin-43 and functional gap junction coupling in human glimas. Glia 33(2): 107-117.
103. Aronica E, Gorter JA, Jansen GH, Leenstra S, Yankava B (2001) Expression of connexin 43 and connexin 32 gap-junction proteins in epilepsy-associated brain tumors and in the perilesional epileptic cortex. Acta Neuropathol 101(5): 449-459.

104. Nakase T, Fushiki S, Naus CC (2003) Astrocytic gap junctions composed of connexin 43 reduce apoptotic neuronal damage in cerebral ischemia. Stroke 34(8): 1987-1993.

105. Nakase T, Fushiki S, Sohl G, Theis M, Willecke K (2003) Neuroprotective role of astrocytic gap junctions in ischemic stroke. Cell Commun Adhes 10(4-6): 413-417.

106. Perez Velazqaue JL, Kokarovtseva L, Sarbaziha R, Jeyapalan Z, Leshchenko (2006) Role of gap junctional coupling in astrocytic networks in the determination of global ischaemia-induced oxidative stress and hippocampal damage. Eur J Neurosci 23(1): 1-10.

107. Castejón OJ. (2013) Increased vesicular and vacuolar transendothelial transport in traumatic human brain oedema. A review Folia Neuropathol 51(2): 93-102.

108. Bellucci A, Zaltieri M, Navarria L, Grigoletto J, Missale C (2012) From $\alpha$-synuclein to synaptic dysfunctions: new insights into the pathophysiology of Parkinson's disease. Brain Res 1476: 183-202.

109. Blanpied TA, Scott DB, Ehlers MD (2002) Dynamic and regulation of clathrin coats as specialized endocytic zones of dendrites and spines. Neuron 36(3): 435-439.

110. Castejón OJ (2009) Blood-brain barrier ultrastructural alterations in human congenital hydrocephalus and Arnold-Chiari malformation. Folia Neuropathol 47(1): 11-19.

111. Castejón OJ (2010) Submicroscopic pathology of human and experimental hydrocephalic cerebral cortex. Folia Neuropathol 48(3): 159-174.

112. Castejón OJ (2011) Ultrastructural pathology of cortical capillary pericytes in human traumatic brain oedema. A Review Folia Neuropathol 49(3): 162-173

113. Cuevas P, Gutierrez Díaz JA, Dujovny M, Diaz FG, Ausman JI (1988) Freeze-fracture cytochemistry of cholesterol content in neuronal plasma membrane following cerebral ischaemia. Neurol Res 10(1): $2-6$.

114. Erb DE, Povlishock JT (1988) Axonal damage in severe traumatic brain injury: an experimental study in cat. Acta Neuropathol (Berl) 76(4): 347-358.

115. Paschen W (1996) Glutamate excitotoxicity in transient global cerebral ischemia. Acta Neurobiol Exp (Wars) 56(1): 313-322.

116. Paschen W, Doutheil J (1999) Disturbance of endoplasmic reticulum functions: A key mechanism underlying cell damage?

117. Paschen W (2003) Endoplasmic reticulum: a primary target in various acute disorders and degenerative diseases of the brain. Cell Calcium 34(4-5): 365-383.

118. Paillusson S, Gomez-Suaga P, Stoica R, Little D, Gissen P et al. (2017) $\alpha$-Synuclein binds to the ER-mitochondria tethering protein VAPB to disrupt $\mathrm{Ca} 2+$ homeostasis and mitochondrial ATP production. Acta Neuropathol 134(1): 129-149.

119. Shi R (2004) The dynamics of axolemmal disruption in guinea pig spinal cord following compression. J Neurocytol 33(2): 203-211.

120. Torp R, Head E, Milgram NW, Hahn F, Otersen OP et al. (2000) Ultrastructural evidence of fibrillar beta-amyloid associated with neuronal membranes in behaviorally characterized aged dog brains. Neuroscience 96(3): 495-506.

121. Walker AK, Atkin JD (2011) Stress signaling from the endoplasmic reticulum: A central player in the pathogenesis of amyotrophic lateral sclerosis. IUBMB Life 63(9): 754-63. 
122. Waxman SG, Black JA, Duncan ID (1990) Macromolecular structure of axon membrane and action potential conduction in melin deficient and myelin deficient heterozygote rat optic nerves. J Neurocytol 19(1): 11-28.

123. Yamaguchi H, Maat-Schieman ML, van Duinen SG, Prins FA, Neeskens $P$ et al. (2000) Amyloid beta protein (Abeta) starts to deposit as plasma membrane-bound form in difuse plaques of brains from hereditary

ISSN: 2574-1241

DOI: 10.26717/BJSTR.2018.10.001988

Orlando J Castejon. Biomed J Sci \& Tech Res

(C) This work is licensed under Creative

Submission Link: https://biomedres.us/submit-manuscript.php cerebral hemorrhage with amyloidosis-Dutch type, Alzheimer disease and nondemented aged subjects. J Neuropathol Exp Neurol 59(8): 105-106.

124. Yi HH, Hoover R, McIntosh T, Hazell AS (2006) Early, transient increase in complexin I and complexin II in the cerebral cortex following traumatic brain injury is attenuated by $\mathrm{N}$-acetylcysteine. J Neurotrauma 23(1): 86-96.

$\begin{array}{ll}\text { BIOMEDICAL } & \text { Assets of Publishing with us } \\ \text { RESEARCHES } & \text { - Global archiving of articles } \\ & \text { - Immediate, unrestricted online access } \\ & \text { - Rigorous Peer Review Process } \\ \end{array}$

